\title{
Can NPT be used to distinguish eosinophilic inflammatory phenotypes in COPD?
}

Hambleton, K., Borg, C., Connolly, C., Bafadhel, M.

Nuffield Department of Clinical Medicine, University of Oxford

Background: Near-patient testing (NPT) is increasingly being used in the management of chronic conditions.[1] In COPD, NPT devices include the spirometer and saturation probe, but these do not directly guide treatment. The blood eosinophil count at a cut off of $\geq 2 \%$ has been shown to determine which patients should receive corticosteroids for exacerbations. [2] An NPT that accurately correlates with whole blood eosinophil counts would be useful.

Methods: We conducted an independent comparison of an NPT device: the HemoCue $\bigcirc$ WBC DIFF against corresponding automated results on 19 volunteers. We compared NPT to automated counts. Samples were taken simultaneously; the NPT was carried out immediately and whole blood was sent for laboratory analysis. Intraclass correlation coefficients (ICC), sensitivity and specificity were calculated, with stratification for allergy status.

Results: The median (range) age was 27 (22-44) years, 7 (37\%) were male and $8(42 \%)$ had a history of allergy. Strong correlations were seen between the HemoCue $\subset$ WBC DIFF and whole blood for white cell count, neutrophils and eosinophils respectively ( $r=0.87 ; r=0.94 ; r=0.71, p<0.01$ for all). The NPT correctly identified a blood eosinophil level of $\geq 2 \%$ with a sensitivity $(95 \% \mathrm{Cl})$ of $100 \%(59-100)$ and a specificity of $83 \%$ (52-98). The mean ICC was 0.89 (0.71-0.96), indicating minimal variability, and was not affected by atopy status.

Conclusion: The HemoCue $\subseteq$ WBC DIFF NPT could be used to guide treatment of COPD exacerbations.

\section{References}

1. Crook, M.A., Near patient testing and pathology in the new millennium. Journal of Clinical Pathology, 2000. 53(1): p. 27-30.

2. Bafadhel, M., et al., Blood eosinophils to direct corticosteroid treatment of exacerbations of chronic obstructive pulmonary disease: a randomized placebo-controlled trial. Am J Respir Crit Care Med, 2012. 186(1): p. 48-55. 